\title{
Synthesis and Biological Evaluation of Novel Fluorine-Containing Stilbene \\ Derivatives as Fungicidal Agents against Phytopathogenic Fungi
}

\author{
Weilin Jian, ${ }^{\dagger}$ Daohang He, ${ }^{*},{ }^{\dagger}$ Pinggen $\mathrm{Xi}^{\ddagger}$ and Xinwei $\mathrm{Li}^{\dagger}$ \\ $\dagger$ School of Chemistry and Chemical Engineering, South China University of \\ Technology, Guangzhou, Guangdong 510640, People's Republic of China \\ * Guangdong Province Key Laboratory of Microbial Signals and Disease Control, \\ South China Agricultural University, Guangzhou, Guangdong 510642, People's \\ Republic of China \\ *Corresponding author: \\ Phone/fax: + 86-20- 8711-0234. \\ E-mail: he16221@163.com or cehdh@scut.edu.cn.
}

Contents

General synthetic procedures and characterization data for key intermediates. S2

References. S4 
General Synthetic Procedures and Characterization Data for Key Intermediates.

The desired starting materials were prepared by the condensation of 4-methylbenzohydrazide and 4-fluorobenzaldehyde in an ethanol solution. ${ }^{1}$ Intermediate $\mathbf{1}$ was synthesized according to our previously reported procedure (Figure S1). ${ }^{1-3}$
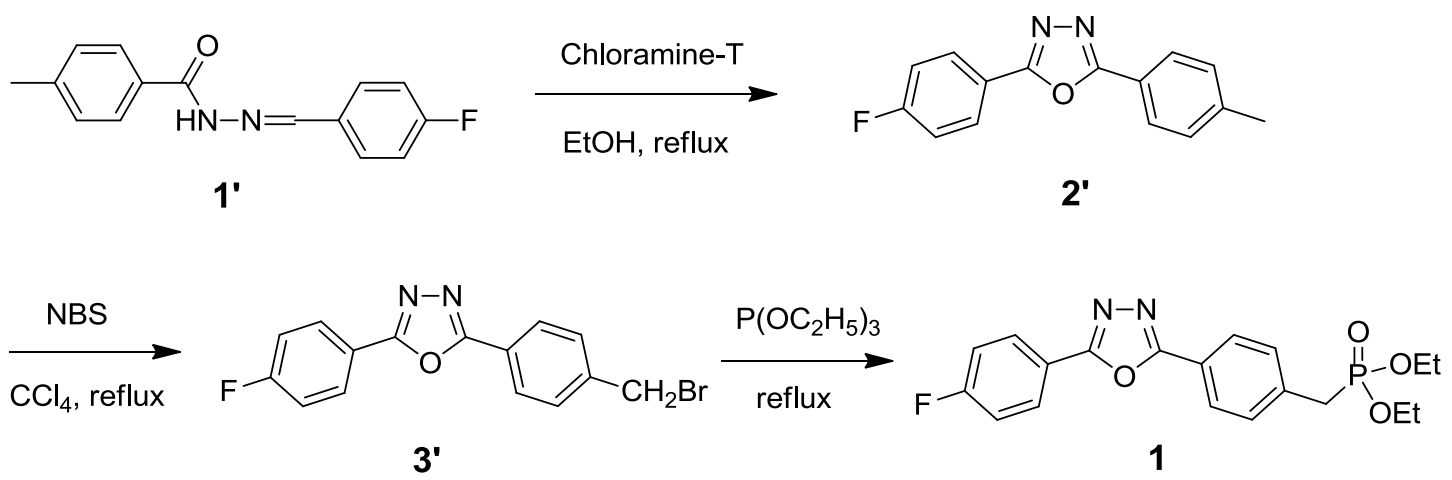

Figure S1. General synthetic route for compound 1.

Synthesis of 2-(4-fluorophenyl)-5-(p-tolyl)-1,3,4-oxadiazole 2'. To a stirred solution of $\mathbf{1}^{\prime}(12.81 \mathrm{~g}, 50 \mathrm{mmol})$ in ethanol $(300 \mathrm{~mL})$ was added Chloramine-T $(42.25 \mathrm{~g}$, $150 \mathrm{mmol})$, the mixture was stirred for $3.5 \mathrm{~h}$ at $75^{\circ} \mathrm{C}$. After the excess ethanol was evaporated, the mixture was washed with water and filtered. The residue was recrystallized from acetone to give compound 2' as white crystals; yield, 68.6\%; mp, $162-163{ }^{\circ} \mathrm{C} ;{ }^{1} \mathrm{H}$ NMR $\left(600 \mathrm{MHz}, \mathrm{CDCl}_{3}\right) \delta 8.16-8.11\left(\mathrm{~m}, 2 \mathrm{H}, \mathrm{C}_{6} \mathrm{H}_{4} 2,6-\mathrm{H}\right), 8.00(\mathrm{~d}$, $\left.J=8.1 \mathrm{~Hz}, 2 \mathrm{H}, \mathrm{C}_{6} \mathrm{H}_{4} 2,6-\mathrm{H}\right), 7.33\left(\mathrm{~d}, J=8.0 \mathrm{~Hz}, 2 \mathrm{H}, \mathrm{C}_{6} \mathrm{H}_{4} 3,5-\mathrm{H}\right), 7.22(\mathrm{t}, J=8.5 \mathrm{~Hz}$, $\left.2 \mathrm{H}, \mathrm{C}_{6} \mathrm{H}_{4} 3,5-\mathrm{H}\right), 2.44\left(\mathrm{~s}, 3 \mathrm{H}, \mathrm{CH}_{3}\right) ;{ }^{13} \mathrm{C} \mathrm{NMR}\left(151 \mathrm{MHz}, \mathrm{CDCl}_{3}\right) \delta$ 165.57, 164.75, $163.89,163.51,142.33,129.77,129.15,129.09,126.85,121.07,120.42,120.40$, 116.42, 116.28, 21.59; MS (ESI), $m / z[\mathrm{M}+\mathrm{Na}]^{+} 278.63$. 
Synthesis of 2-(4-(bromomethyl)phenyl)-5-(4-fluorophenyl)-1,3,4-oxadiazole 3'. To a stirred solution of $\mathbf{2}^{\prime}(12.71 \mathrm{~g}, 50 \mathrm{mmol})$ in carbon tetrachloride $(300 \mathrm{~mL})$ was added N-Bromosuccinimide (NBS) (10.32 g, $58 \mathrm{mmol}$ ), the reaction was heated under reflux for $8 \mathrm{~h}$ and then the excess solvent was removed under reduced pressure. The resulting mixture was filtered, washed with ethanol. The residue was recrystallized from THF/ethanol to give compound $\mathbf{3}^{\prime}$ as white solids; yield, $62.6 \%$; mp, 172 $173{ }^{\circ} \mathrm{C} ;{ }^{1} \mathrm{H}$ NMR $\left(600 \mathrm{MHz}, \mathrm{CDCl}_{3}\right) \delta 8.15\left(\mathrm{dd}, J=7.6,5.3 \mathrm{~Hz}, 2 \mathrm{H}, \mathrm{C}_{6} \mathrm{H}_{4} 2,6-\mathrm{H}\right)$, $8.11\left(\mathrm{~d}, J=7.2 \mathrm{~Hz}, 2 \mathrm{H}, \mathrm{C}_{6} \mathrm{H}_{4} 2,6-\mathrm{H}\right), 7.57\left(\mathrm{~d}, J=7.8 \mathrm{~Hz}, 2 \mathrm{H}, \mathrm{C}_{6} \mathrm{H}_{4} 3,5-\mathrm{H}\right), 7.24(\mathrm{t}, J$ $\left.=8.1 \mathrm{~Hz}, 2 \mathrm{H}, \mathrm{C}_{6} \mathrm{H}_{4} 3,5-\mathrm{H}\right), 4.54\left(\mathrm{~s}, 2 \mathrm{H}, \mathrm{CH}_{2}\right) ;{ }^{13} \mathrm{C} \mathrm{NMR}\left(151 \mathrm{MHz}, \mathrm{CDCl}_{3}\right) \delta 165.70$, $164.12,164.02,163.88,141.55,129.75,129.28,129.22,127.32,123.73,120.21$, 120.19, 116.53, 116.38, 32.16; MS (ESI), $m / z . \mathrm{M}+\mathrm{Na}]^{+} 356.06$.

Synthesis of diethyl 4-(5-(4-fluorophenyl)-1,3,4-oxadiazol-2-yl) benzylphosphonate 1. A mixture of compound 3' (10.00 g, $30 \mathrm{mmol})$ and triethyl phosphite $(50 \mathrm{~mL})$ was heated under reflux for $5 \mathrm{~h}$. The excess triethyl phosphate was evaporated under reduced pressure, and then filtered by addition of hexane. Recrystallization from THF/hexane afforded compound $\mathbf{1}$ as white solids, yield, 85.3\%; mp, $104-105{ }^{\circ} \mathrm{C} ;{ }^{1} \mathrm{H}$ NMR (600 MHz, $\left.\mathrm{CDCl}_{3}\right) \delta 8.13\left(\mathrm{~m}, 2 \mathrm{H}, \mathrm{C}_{6} \mathrm{H}_{4} 2,6-\mathrm{H}\right)$, $8.07\left(\mathrm{~d}, J=8.1 \mathrm{~Hz}, 2 \mathrm{H}, \mathrm{C}_{6} \mathrm{H}_{4} 2,6-\mathrm{H}\right), 7.48\left(\mathrm{dd}, J=8.3,2.3 \mathrm{~Hz}, 2 \mathrm{H}, \mathrm{C}_{6} \mathrm{H}_{4} 3,5-\mathrm{H}\right), 7.22$ $\left(\mathrm{m}, 2 \mathrm{H}, \mathrm{C}_{6} \mathrm{H}_{4} 3,5-\mathrm{H}\right), 4.05\left(\mathrm{~m}, 4 \mathrm{H}, \mathrm{OCH}_{2}\right), 3.23\left(\mathrm{~d}, J=22.1 \mathrm{~Hz}, 2 \mathrm{H}, \mathrm{CH}_{2}\right), 1.26(\mathrm{t}, J=$ 7.1 Hz, 6H, $\left.\mathrm{CH}_{3}\right) ;{ }^{13} \mathrm{C} \mathrm{NMR}\left(151 \mathrm{MHz}, \mathrm{CDCl}_{3}\right) \delta 165.63,164.42,163.95,163.72$, $136.13,136.07,130.52,129.20,129.15,127.05,122.46,120.29,120.27,116.47$, $116.32,62.30,62.25,34.47,33.56,16.36,16.32 ; \mathrm{MS}(\mathrm{ESI}), \mathrm{m} / z[\mathrm{M}+\mathrm{Na}]^{+} 413.94$. $^{+}$ 


\section{References}

(1) Li, X. W.; He, D. H. Synthesis and optical properties of novel anthracene-based stilbene derivatives containing an 1,3,4-oxadiazole unit. Dyes Pigm. 2012, 93, $1422-1427$.

(2) Lu, H. X.; He, D. H. Asymmetric 1,3,4-oxadiazole derivatives containing naphthalene and stilbene units: synthesis, optical and electrochemical properties. Spectrochim. Acta, Part. A 2014, 124, 91-96.

(3) Li, X. W.; Lu, H. X.; He, D. H.; Luo, C.; Huang, J. J. Synthesis, fluorescence properties and theoretical calculations of novel stilbene derivatives based on 1,3,4-oxadiazole bearing anthracene core. J. Fluoresc. 2013, 23, 1039-1044. 